\title{
AN UPPER BOUND FOR THE JOHN CONSTANT
}

\author{
JULIAN GEVIRTZ
}

Abstract. A new upper bound for the John constant is established.

Let $F$ denote the set of functions $f(z)$ which are nonconstant and analytic in the unit disk $|z|<1$ and let $G$ denote the subset of $F$ consisting of those $f(z)$ in $F$ which are not univalent. For $f(z)$ in $F$ we define

$$
M(f)=\sup _{|z|<1}\left|f^{\prime}(z)\right| \text { and } m(f)=\inf _{|z|<1}\left|f^{\prime}(z)\right| .
$$

John [1] defined the constant $\gamma$ by

$$
\gamma=\inf _{f \in G} \frac{M(f)}{m(f)} .
$$

This constant has come to be known as the John constant. In the same paper John showed that $\gamma \geqslant \exp (\pi / 2)=4.810 \ldots$ and also established an upper bound for $\gamma$ which was later improved by Yamashita [2] who showed that $\gamma<\exp (\pi)=$ $23.140 \ldots$. The purpose of this note is to improve this upper bound further. We will show that $\gamma \leqslant \exp (\lambda \pi)$, where $\lambda$ is the solution to a certain transcendental equation. Numerical calculations show that $.6278<\lambda<.6279$ and this yields $\gamma<\exp (.6279 \pi)=7.189 \ldots$

We work in the upper half-plane $\operatorname{Im} z>0$ instead of in the disk. In what follows, $H$ and $\bar{H}$ will denote the open and closed upper half-plane, respectively. Let $f(z)$ be analytic and not univalent in $H$. If $\alpha$ and $\beta$ denote the infimum and supremum of $\left|f^{\prime}(z)\right|$ in $H$, respectively, then $\gamma \leqslant \alpha / \beta$. To see this we note that since $f(z)$ is not univalent in $H$, it must be nonunivalent in some disk $|z-c|<r$ contained in $H$. But then $g(z)=f(c+r z)$ belongs to $G$ and $M(g)<r \beta, m(g)>r \alpha$ and consequently $\gamma \leqslant \beta / \alpha$.

For the nonunivalent function $f(z)$ which we study here $\left|f^{\prime}(z)\right|$ is piecewise constant on the real line. What is more, it is the simplest kind of nontrivial piecewise constant function, namely one that takes on one constant value on some interval and another outside that interval. Our choice of $f(z)$ is motivated by the following consideration: Let $z_{1} \neq z_{2}$ be two points in $|z|<1$. One can show using standard variational arguments that if $h_{0}(z)$ is a function for which $\left|h\left(z_{1}\right)-h\left(z_{2}\right)\right|$ attains a positive minimum for the class of functions $h(z)$ which satisfy $m<\left|h^{\prime}(z)\right|$ $\leqslant M$ in $|z|<1$, then $\left|h_{0}^{\prime}(z)\right|$ is piecewise constant on $|z|=1$. (This fact is solely

Received by the editors November 3, 1980 and, in revised form, March 24, 1981.

1980 Mathematics Subject Classification. Primary 30A40.

Key words and phrases. John constant. 
mentioned as motivation; it is not used in what follows.) Since for convenience we choose to work in $H$, we are led to examine the analogue in $H$ of the simplest nontrivial case of such a function. Indeed, these considerations suggest the possibility that our upper bound of $\exp (\lambda \pi)$ is the true value of $\gamma$.

We denote by $\log z$ the principal branch of $\log z$; that is, $\log z=\log |z|+$ $i \operatorname{Arg} z$, where $0 \leqslant \operatorname{Arg} z<2 \pi$. Let $k(z)=\exp (\mu i(\log (z-1)-\log (z+1)))$ where $\mu>0$. We note in passing that $k(z)$ is single valued in the complement of the real interval $[-1,1]$, and that \pm 1 are its only singularities (branch points). Since $|k(z)|=\exp (-\mu(\operatorname{Arg}(z-1)-\operatorname{Arg}(z+1)))$, one has that for $z$ in $\bar{H} \backslash\{-1,1\}$, $\exp (-\mu \pi) \leqslant|k(z)| \leqslant 1$, and that for $x$ real $|k(x)|$ is equal to 1 if $|x|>1$ and is equal to $\exp (-\mu \pi)$ if $|x|<1$. Let

$$
f(z)=\int_{0}^{z} k(w) d w, \quad \text { for } z \in \bar{H} .
$$

Here the path of integration may be taken to be the straight line segment between 0 and $z$, even when this segment contains 1 or -1 , since the integrand is bounded in $\bar{H}$. Let $a>1$ be defined by $\mu \log ((a-1) /(a+1))=-\pi / 2$. We shall evaluate

$$
f(a)-f(-a)=\int_{-a}^{a} k(z) d z
$$

where we integrate along the segment $[-a, a]$. Let $I_{1}=\int_{-1}^{1} k(t) d t . I_{1}$ may be easily evaluated by the following argument: As noted above, $k(z)$ is analytic and single valued in the complement of $[-1,1]$. The argument of $k(z)$ at any point $t$ of the slit $[-1,1]$ is the same on the top of this slit as on the bottom. However, on the top $|k(t)|$ is $\exp (-\mu \pi)$ and on the bottom it is $\exp (\mu \pi)$. Thus, by the Cauchy integral theorem,

$$
I_{1}(\exp (2 \mu \pi)-1)=\int_{C_{R}} k(z) d z
$$

where $C_{R}$ is the circle $|z|=R>1$, oriented positively. It is not difficult to see that $k(z)=1-2 \mu i / z+O\left(1 /|z|^{2}\right)$. Thus, $I_{1}(\exp (2 \mu \pi)-1)=4 \mu \pi+O(1 / R)$, which implies that $I_{1}=4 \mu \pi /(\exp (2 \mu \pi)-1)$.

Now let

$$
I_{2}(a)=\int_{-a}^{-1} k(t) d t+\int_{1}^{a} k(t) d t=2 \int_{1}^{a} \cos \left(\mu \log \frac{t-1}{t+1}\right) d t .
$$

After making the substitution $s=\log ((t-1) /(t+1))$, simple computations show that

$$
I_{2}(a)=\int_{\pi / 2 \mu}^{\infty} \frac{\cos \mu s}{\sinh ^{2}(s / 2)} d s .
$$

We have $1 / \sinh ^{2}(s / 2)=4 e^{-s} /\left(1-e^{-s}\right)^{2}=4 \sum_{n=1}^{\infty} n e^{-n s}$, and consequently,

$$
I_{2}(a)=4 \int_{\pi / 2 \mu}^{\infty} \sum_{n=1}^{\infty} n e^{-n s} \cos \mu s d s,
$$

which may be integrated term by term (by dominated convergence, for example) yielding

$$
I_{2}(a)=-4 \mu \sum_{n=1}^{\infty} \frac{n}{n^{2}+\mu^{2}} \exp \left(-\frac{n \pi}{2 \mu}\right)
$$


We thus conclude that

$$
f(a)-f(-a)=\frac{4 \mu \pi}{e^{2 \mu \pi}-1}-4 \mu \sum_{n=1}^{\infty} \frac{n}{n^{2}+\mu^{2}} \exp \left(-\frac{n \pi}{2 \mu}\right) .
$$

Let $\phi(\mu)$ denote the expression on the right-hand side of this last equation. Let $\mu>0$ be such that $\phi(\mu)<0$. For this value of $\mu$ and for any $b>a$,

$$
I_{2}(b)=I_{2}(a)+2 \int_{a}^{b} \cos \left(\mu \log \frac{t-1}{t+1}\right) d t .
$$

On the interval $[a, \infty)$ the function $\mu \log ((t-1) /(t+1))$ varies monotonically from $-\pi / 2$ to 0 , so that on this interval the function $\cos (\mu \log ((t-1) /(t+1)))$ is positive and tends to 1 as $t$ tends to infinity. Since

$$
f(b)-f(-b)=\phi(\mu)+2 \int_{a}^{b} \cos \left(\mu \log \frac{t-1}{t+1}\right) d t,
$$

there is a $b>a$ for which $f(b)-f(-b)=0$. However,

$$
\operatorname{Arg} f^{\prime}(b)=\mu \log \left(\frac{b-1}{b+1}\right) \in\left(-\frac{\pi}{2,0}\right)
$$

and similarly, $\operatorname{Arg} f^{\prime}(-b) \in(0, \pi / 2)$. Since $f(z)$ is conformal at $\pm b$ and since by what we have just shown $f^{\prime}(b) / f^{\prime}(-b)$ is not negative, simple geometric considerations show that there must be points $p$ and $q$ arbitrarily close to $b$ and $-b$, respectively, and lying in $H$ such that $f(p)=f(q)$. Thus $f(z)$ is not univalent in $H$.

To finish, we must examine $\phi(\mu)$. It is a simple matter to establish numerically that there is a $\lambda$ in $(.6278, .6279)$ for which $\phi(\lambda)=0$ and $\phi^{\prime}(\lambda)<0$. Hence, for any $\varepsilon>0$ there is a $\mu$ in $(\lambda, \lambda+\varepsilon)$ such that $\phi(\mu)<0$. But this means that there is an $f(z)$ analytic and not univalent in $H$ for which $M(f) / m(f)=\exp (\mu \pi)$. Consequently, $\gamma \leqslant \exp (\lambda \pi)<\exp (.6279 \pi)=7.189 \ldots$

\section{REFERENCES}

1. F. John, A criterion for univalence brought up to date, Comm. Pure Appl. Math. 29 (1976), 293-295.

2. S. Yamashita, On the John constant, Math. Z. 161 (1978), 185-188.

Instrtuto de Matemática, Ponttficia Universidad Católica de Chme, Casilla 114-D, Santiago, CHILE 